\title{
An Optimization Model of Multi-Intersection Signal Control for Trunk Road under Collaborative Information
}

\author{
Xun Li, ${ }^{1}$ Zhengfan Zhao, ${ }^{2}$ Li Liu, ${ }^{3}$ Yao Liu, ${ }^{1}$ and Pengfei Li ${ }^{1}$ \\ ${ }^{1}$ College of Electronics and Information, Xian Polytechnic University, Xian 710048, China \\ ${ }^{2}$ Beijing Special Electromechanical Technology Research Institute, Beijing 100012, China \\ ${ }^{3}$ College of Management, Xian Polytechnic University, Xian 710048, China \\ Correspondence should be addressed to Xun Li; upintheair037@163.com
}

Received 18 January 2017; Revised 13 April 2017; Accepted 19 April 2017; Published 25 May 2017

Academic Editor: Enrique Onieva

Copyright (c) 2017 Xun Li et al. This is an open access article distributed under the Creative Commons Attribution License, which permits unrestricted use, distribution, and reproduction in any medium, provided the original work is properly cited.

\begin{abstract}
We proposed a signal control optimization model for urban main trunk line intersections. Four-phase intersection was analyzed and modeled based on the Cell Transmission Model (CTM). CTM and signal control model in our study had both been improved for multi-intersections by three-phase theory and information-exchanging. To achieve a real-time application, an improved genetic algorithm (GA) was proposed finally, the DISCO traffic simulation software was used for numerical simulation experiment, and comparisons with the standard GA and CTM were reported in this paper. Experimental results indicate that our searching time is less than that of SGA by $38 \%$, and our method needs only $1 / 3$ iteration time of SGA. According to our DISCO traffic simulation processing, compared with SGA, if the input traffic flow is changed from free phase to synchronized phase, for example, less than $900 \mathrm{vel} / \mathrm{h}$, the delay time can reduce to $87.99 \%$ by our method, and the minimum delay time is $77.76 \%$ of existing method. Furthermore, if input traffic volume is increased to $1200 \mathrm{vel} / \mathrm{h}$ or more at the synchronized phase, the summary and minimum values of average delay time are reduced to $81.16 \%$ and $75.83 \%$, respectively, and the average delay time is reduced to 17.72 seconds.
\end{abstract}

\section{Introduction}

Multi-intersection signal cooperative control is one way to solve the urban congestion problem, especially at 3D urban trunk line intersections which are difficult to upgrade. As one solution, cooperative method in traffic control has been studied in purpose to solve the problem. There are many typical control systems for road network [1], for example, TRANSYT-7F, SCAT system, traffic control system of Kyosan company and Siemens, and the STC traffic control systems in China. All these systems have the ability to time traffic signals according to traffic flow both upstream and downstream. However, these systems are not designed for saturation flow, resulting in a possible lack of performance under serious traffic jam [2].

As one solution of intersection optimization control under traffic congestion situation, genetic algorithm (GA) was used to solve the problem by Park et al. [3]. GA improves an early research [4] that only can generate optimization control signals under unsaturated state. In addition, GA also takes into account network delay and road capacity. However, the optimization process in GA requires the assumption that the state of traffic flow is stable over a period of time. Lieberman and Chang [5] use the queue length of each intersection as constraint and propose the Mixed Integer Linear Programming (MILP) to solve the optimal signal phase difference, which can adjust the green time in a signal cycle by nonlinear quadratic programming. Abu-Lebdeh and Benekohal [6] also use queue length as the object of the management, whose performance of dynamic control is evaluated based on negative utility function. Then, they use GA to find the optimal value. One drawback of previous researches is that several important pieces of information are not taken into account, such as the relationship between adjacent vehicles, or neglect the traffic flow dynamics. So these schemes may fail to cover the unsaturated, saturated, or supersaturated state of traffic flow.

Cell Transmission Model (CTM) is a model which approximates the macroscopic dynamic model LWR [7] as discretization function for describing continuous traffic flow. 
Based on this feature, Lo and his research team [8] proposed a new sensitivity analysis method for dynamic signal control using the physical characteristics of vehicle; Zhengwu et al. [9] studied the traffic flow at the entrance approach road of the intersection and proposed an improved method of the cell division and its state description based on CTM. Yunfeng et al. [10] analyzed the influence of VMS on two traffic conditions: repetitive and nonrepetitive congestion. A multiobjective optimization model of traffic signal coordinated control parameters is established. They solve a multiobjective optimization problem based on nondominated sorting genetic algorithm. However, the mentioned researches solved problems by the standard CTM as the basic model; a method ignored the influence of three-phase traffic flow [11, 12].

In summary, CTM can cover the whole range of flowdensity-velocity in signal control synergy and clearly describe the physical effect of queue. Therefore, in this paper, an intersection traffic flow model was proposed based on the traffic dynamics model by the improved CTM, which is promoted by the three-phase theory under different traffic flow characteristics. In order to solve the problems of multi-intersection signal optimization parameters in real-time, an improved genetic algorithm is used to optimize the parameters. Finally, we carried out experiments according to one real-world traffic data, captured at "Bianjiacun, Nanshaomeng" main trunk line of Xi'an Youyi Road, to verify the performance of our method. The results show that the proposed method can improve the timing scheme in many traffic situations. It is an effective signal optimization control strategy based on information interaction.

\section{CTM Model for Multi-Intersection Signal Control}

2.1. Four-Phase Intersection Model Based on CTM. LWR model of traffic flow is simplified by CTM [13], which gives a finite difference solution. Recursion formula of conservation equation transformation CTM can be expressed by formulas (1) and (2).

$$
\begin{aligned}
& n_{i}(t+1)=n_{i}(t)+f_{i}(t)-f_{i+1}(t) \\
& f_{i}(t)=\min \left(n_{i-1}(t), Q_{i}(t),\left(\frac{\omega}{v_{f}}\right)\left(N_{i}(t)-n_{i}(t)\right)\right) .
\end{aligned}
$$

The subscript $i$ means cell ordinal number and $i \pm 1$ are up- or downstream cells. Formula (1) is the traffic flow value of the $i$ cell at time $t+1$, which equals the traffic flow value about the sum of $i$ cell and the inflow $f_{i}(t)$ and outflow $f_{i+1}(t)$, inflow $f_{i}(t)$ is the traffic value flow into the cell $i$ at time $t$, and outflow $f_{i+1}(t)$ is the traffic value flow out of the cell $i$ at the same time. Formula (2) means the input capacity of $i$ cell is restricted by its own capacity $Q_{i}(t)$ and upstream traffic cell value $n_{i-1}(t)$ and currently accessible traffic value at time $t$, where $N_{i}(t)$ is the maximum allowable traffic of cell $i$ at time $t, \omega$ is the backpropagation velocity of crowded perturbation, and $v_{f}$ is the speed of free traffic flow.

According to the four-phase signal control in the main trunk line in the urban traffic environment, the traffic flow

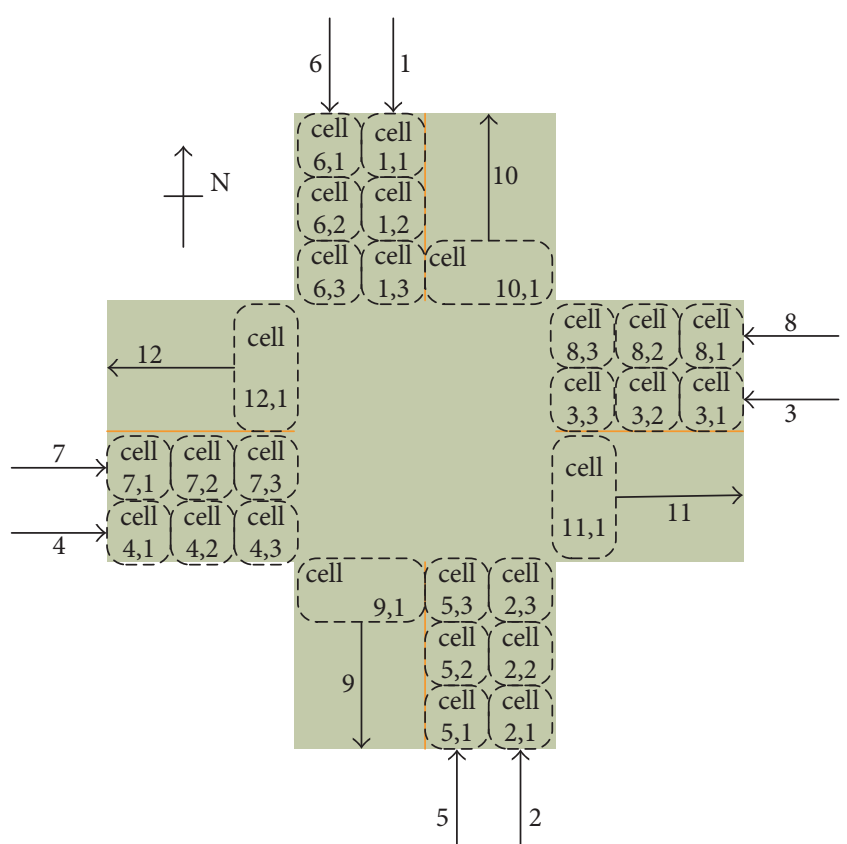

FIGURE 1: Schematic diagram of the single intersection four-phase CTM mode.

CTM is established as Figure 1: cell ${ }_{i j}$ represents the $j$ cell of the $i$ traffic flow. For each cell, $N_{i j}(t)$ represents the capacity of vehicles at time $t$. The traffic flow can be divided into two independent types as source stream and the exit stream according to the state of the intersection [14]. Meanwhile, in the four-phase control model, the right-turn vehicles are not controlled by signal; the CTM can be described as follows.

(i) Source Cell cell ${ }_{i 1}$. The 1st cell in each source is described as the amount of traffic $n_{i 1}^{k}(t)$, which is flow into the road. Suppose upstream is still a four-phase controlled intersection; $n_{i 1}^{k}(t), k=1,2, \ldots, 8$, can be acquired by function (3), where $d_{i}^{k-1}(t)$ is the component traffic flow of $i$ traffic flow corresponding to the $k$ intersection, which is from the upstream intersection $k-1$ flow.

$$
n_{i 1}^{k}(t)=\sum_{k} d_{i}^{k-1}(t), \quad i=k=1,2, \ldots, 8
$$

(ii) Source Cell cell ${ }_{i 2}$. The capacity of the current road can be obtained by function (4).

$$
Q_{i 2}(t)= \begin{cases}Q_{i 2 \cdot \max }-N_{i 2}(t)+Q_{i 3 . g}(t) & \text { green } \\ Q_{i 2 \cdot \max }-N_{i 2}(t) & \text { red, }\end{cases}
$$

where $Q_{i 2 \text {.max }}$ is saturated flow of the $i$ traffic flow; and $N_{i 2}(t)$ is the traffic value in the present; $Q_{i 3 . g}(t)$ is the outflow of traffic, when the corresponding traffic is at green state. 
(iii) Source Cell cell ${ }_{i 3}$. The third source cell simulates signal control.

$$
\begin{aligned}
& Q_{i 3 . g}(t)=\min \left(F_{i . v f}(t), Q_{i . o u t}(t+1)\right) \text { green, } \\
& Q_{i 3 . r}(t)=0 \quad \text { red. }
\end{aligned}
$$

In function (5), at the time of green light, the maximum traffic flow is corresponding to traffic free speed flow volume $F_{i . v f}(t)$ and the outlet flow cell capacity $Q_{i . \text { out }}(t+1)$.

(iv) Export Cell. According to the mathematical description of the source cells, the export cell capacity is related to the capacity downstream and the traffic component flow upstream. Hence, the capacity of the export flow cell can be expressed by

$$
Q_{i . \text { out }}(t)=\min \left(n_{i 1}^{k}(t), Q_{i .2}(t)\right)
$$

2.2. Extended CTM in Traffic Signal Net. When the CTM is applied into multi-intersection signal control, the upstream cells exist as distributary and the downstream cells exist as confluent flow [15].

(i) Direct Transmission. As shown in (7), it is related to upstream incoming traffic and downstream reception capabilities; the symbol is the same as (4).

$$
\begin{aligned}
& f_{i+1}(t)=\min \left(n_{i}(t), Q_{i}(t), Q_{i+1}(t),\left(\frac{\omega}{v_{f}}\right),\right. \\
& \left.\left(N_{i+1}(t)-n_{i+1}(t)\right)\right) .
\end{aligned}
$$

(ii) Separated Transmission. In left-turn phase, split transmission can be regarded as direct transmission; straight and turn right are in the same phases, which have the separated transmission. Suppose the steering cell as cell $i_{c}$, existing in $n_{i_{c}}(t), Q_{i_{c}}(t)$, traffic flow $i_{c}$ transport into cell $i+1$ can be obtained by functions (8) and (9).

$$
\begin{aligned}
& f_{i+1}(t)=\min \left(\left(n_{i}(t), Q_{i}(t)\right)\right. \\
& \left.\cdot p_{i}, Q_{i+1}(t),\left(\frac{\omega}{v_{f}}\right)\left(N_{i+1}(t)-n_{i+1}(t)\right)\right), \\
& f_{i_{c}}(t)=\min \left(\left(n_{i}(t), Q_{i}(t)\right)\right. \\
& \left.\cdot p_{c}, Q_{i_{c}}(t),\left(\frac{\omega}{v_{f}}\right)\left(N_{i_{c}}(t)-n_{i_{c}}(t)\right)\right),
\end{aligned}
$$

where $p_{i}=f_{i+1}(t) / f_{i}(t), p_{c}=f_{i_{c}}(t) / f_{i}(t), p_{i}+p_{c}=1$ is the ratio of the straight and turn-left traffic flow from export cell $i$, and $f_{i_{c}}(t)+f_{i+1}(t)$ is the total output traffic volume. (iii) Confluence Transmission. When the upstream input is less than the downstream input traffic, the traffic flow into cell $i+1$ can be given by function (10):

$$
\begin{gathered}
f_{i+1}(t)=\min \left(\left(n_{i}(t), Q_{i}(t)\right) \cdot p_{i}\right) \\
+\min \left(\left(n_{i}(t), Q_{i_{c}}(t)\right) \cdot p_{c}\right) .
\end{gathered}
$$

When the downstream input is less than the upstream input traffic, the traffic output from cell $i$ and steering cell $i_{c}$ can be given by function (11):

$$
\begin{aligned}
& f_{i}(t)=\min \left(n_{i}(t), Q_{i}(t),\left(\left(n_{i+1}(t), Q_{i+1}(t)\right) \cdot p_{i}\right)\right), \\
& f_{i_{c}}(t) \\
& \quad=\min \left(n_{i_{c}}(t), Q_{i_{c}}(t),\left(\left(n_{i+1}(t), Q_{i+1}(t)\right) \cdot p_{c}\right)\right),
\end{aligned}
$$

where $p_{c}+p_{i}=1$ is the ratio of the straight and turn cell traffic value from the export cell $i$. In the simulation, in order to reduce the length of vehicle queuing, the ratio is determined according to the traffic volume of each cell at time $t$, and higher export flow with higher ratio. So $p_{c}, p_{i}$ can be simplified as

$$
\begin{aligned}
& p_{i}=\frac{n_{i}(t)}{\left(n_{i}(t)+n_{i_{c}}(t)\right)}, \\
& p_{c}=1-p_{i} .
\end{aligned}
$$

\section{Improved CTM for Multi-Intersection}

3.1. Improved CTM of One Intersection. Based on the characteristics of directed model, separated model, and confluence model this subsection presents an improved CTM model for trunk traffic flow under signal interaction.

As Youyi Road shows from intersection Bianjiacun to the intersection Zhuque, according to historical data of traffic flow, the east-west direction is the trunk road-West Road, as Figure 2 shows. The simplified model of trunk traffic flow under the condition of road information is shown in Figure 3 based on CTM.

cell $_{i j}^{k}$ is $j$ cell of $i$ traffic flow at $k$ intersection, whose mathematical description is the same as Section 2.2.

Combined with the traffic flow number in Figure 1, taking the $k$ intersection as an example, the traffic flow transfer out from an intersection to the source cell of downstream can be expressed as

$$
\begin{aligned}
& f_{41}^{k+1}(t)+f_{71}^{k+1}(t)=\min \left(\left(n_{13}^{k}(t)+n_{73}^{k}(t)+n_{23}^{k}\right),\right. \\
& \left.\left(Q_{42}^{k+1}(t)+Q_{72}^{k+1}(t)\right)\right),
\end{aligned}
$$

where $f_{i 1}^{k}(t)$ is $j$ cell traffic volume in $i$ traffic source from $k$ intersection at time $t$, and $Q_{i 2}^{k}(t)$ is capacity of $i$ source cell of $k$ intersection at time $t$. If defined $t_{i}^{k}$ is phase time, where $i$ traffic flow passes $k$ intersection at time $t$, and four-phase 


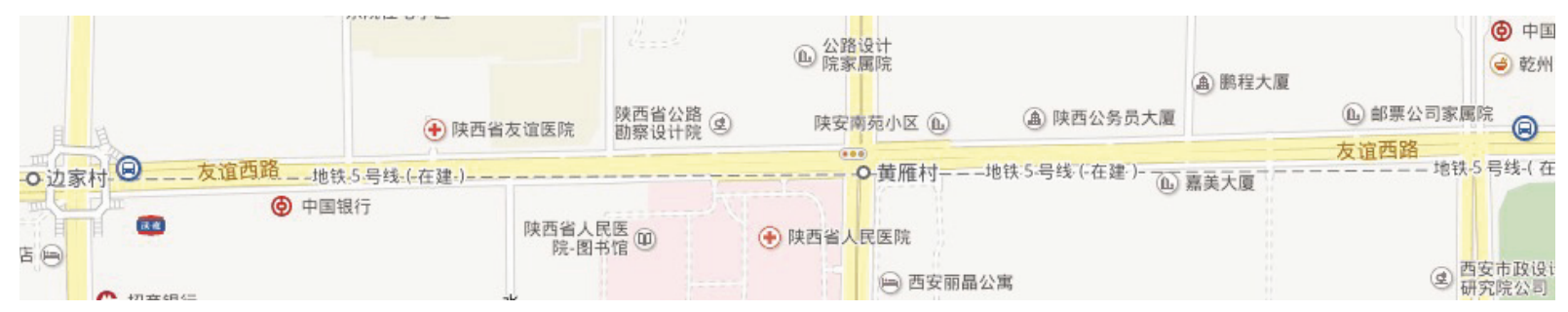

Figure 2: Schematic diagram of three-intersection trunk line.

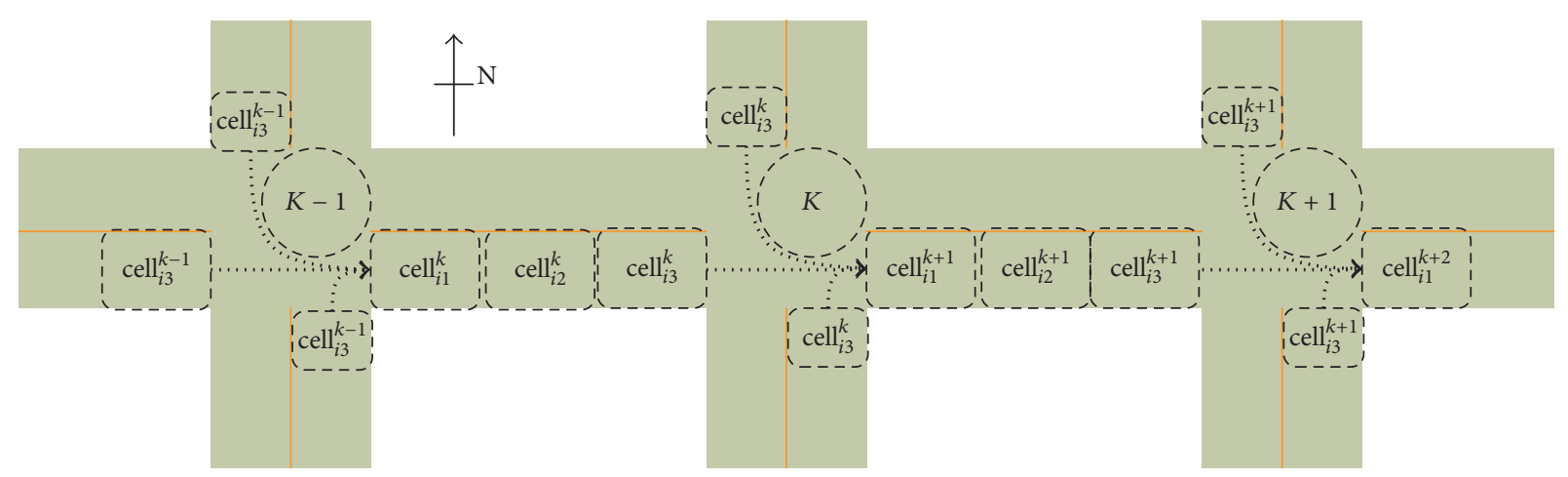

Figure 3: Schematic diagram of intersections traffic flow transmission.

signal cycle time is $T^{k}$, function (13) can be decomposed into (14) for different phase times.

$$
\begin{aligned}
& f_{41}^{k+1}(t)+f_{71}^{k+1}(t) \\
& =\min \left(\left(n_{73}^{k}(t)+n_{23}^{k}(t)\right),\left(Q_{42}^{k+1}(t)+Q_{72}^{k+1}(t)\right)\right), \\
& f_{41}^{k+1}(t)+f_{71}^{k+1}(t) \\
& \quad=\min \left(\left(n_{13}^{k}(t)+n_{23}^{k}(t)\right),\left(Q_{42}^{k+1}(t)+Q_{72}^{k+1}(t)\right)\right) .
\end{aligned}
$$

The traffic flows through intersection $k$ include straight and left-turn traffic flows $i$ over time $t_{i}^{k}$ which can be given by function (15):

$$
n_{i 3}^{k}(t)=\min \left(Q_{i 2}^{k}(t), N_{i . s}^{k}(t), Q_{i 2}^{k+1}(t)\right)
$$

where $N_{i . s}^{k}(t)$ is the capacity of the $k$ intersection for $i$ traffic flow at time $t$, which can be computed by function (16).

$$
N_{i . s}^{k}(t)=3600 \cdot \psi_{s} \frac{\left(\left(t_{i}^{k}-t_{1}\right) / t_{s}+1\right)}{T^{k}}
$$

where $\psi_{s}$ is the capacity reduction factor of straight-line, and $t_{1}$ is the time where the first car starts and passes the stop line, when the green light is turned on, where $t_{s}$ is the average time of straight vehicles passing through the stop line.
In standard CTM, it assumes that all vehicles cross an intersection with the maximum speed of free flow. $t_{s}$ in formula (16) is calculated based on $v_{\text {free }}$, which is described in formula (5); the maximum traffic flow of intersection capacity is corresponding to the traffic free speed flow volume $F_{i, v f}(t)$. However, in reality, there are speed disturbances, and the average speed of traffic flow is related to density of traffic. For this reason, model of green wave (GW) [11] and KernerKlenov stochastic three-phase microscopic traffic flow model [16] are used for improving the standard CTM.

In this paper, we pay more attention to the signal control efficiency under different situations with various traffic flows and vehicle densities. For cooperative vehicle infrastructure system (CVIS), information of signal control timing can be interchanged among each intersection and every vehicle. In order to minimize the commuting time, we assume future traffic in city that

(i) vehicles are moving on road with the maximum speed related to traffic density;

(ii) the probability of random deceleration is zero, that is, car will be controlled automatically and reasonably;

(iii) the crossing speed is the maximum value based on different traffic density;

(iv) waiting car on beginning of green phase is observing the rule of slow starting; car length of simulation is assumed as 5 meters.

Based on intersection model of the three-phase theory of city traffic [11], formula (16) is improved as 


$$
N_{i . s}^{k}(t)= \begin{cases}\frac{1}{v} \int_{0}^{v} q_{i . \mathrm{in}}^{k}(t) d t, & \bar{q}_{\text {in }}<C_{\text {min }}, \text { no GW breakdown } \\ C_{\max } & \bar{q}_{\text {in }}<C_{\max }, \text { certain GW breakdown } \\ \min \left(\frac{1}{v} \int_{0}^{v} q_{i . \mathrm{in}}^{k}(t) d t, C_{\max }\right) & C_{\min }<\bar{q}_{\text {in }}<C_{\max }, \text { probability GW breakdown. }\end{cases}
$$

In formula (17), the speed value of $v$ is related to the density of traffic flow based on Figure 4 [17]; $C_{\max }$ is the maximum signal capacity and $C_{\min }$ is equal to the classical signal capacity [18].

As the calculated above, the amount of traffic $N_{i 2}^{k}(t)$ between the two intersections in a signal controls cycle time $T^{k}$ which can be obtained by

$$
\begin{aligned}
\int_{t}^{t+T^{k}} & N_{i 2}^{k}(t+1) d t \\
= & N_{i 2}^{k}(t)+\int_{t}^{t+T^{k}} n_{i 1}^{k}(t) d t \\
& \quad-\min \left(\left(D_{i 3}^{k}(t)-\int_{t}^{t+t_{i}^{k}} N_{i . s}^{k}(t) d t\right), D_{i 3}^{k}(t)\right),
\end{aligned}
$$

where $D_{i j}^{k}(t)$ is the delay time of the $j$ cell in the $i$ traffic flow at time $t$ and it can be calculated by the method of literature [19].

$$
D_{i j}^{k}(t)=n_{i j}^{k}(t)-f_{i(j+1)}^{k}(t) .
$$

Formula (5) of standard CTM is improved to (20). The capacity of intersection in one green phase is calculated based on the density of traffic flow at the interesting moment.

$$
\begin{aligned}
& Q_{i 3 . g}(t)=\min \left(\int_{t}^{t+T^{k}} N_{i 2}^{k}(t+1) d t, Q_{i . \text { out }}(t+1)\right) \\
& \text { green, } \\
& Q_{i 3 . r}(t)=0 \text { red. }
\end{aligned}
$$

\subsection{Multi-Intersection Signal Control Model of Trunk Road}

\section{(i) Objective Function}

(a) Minimum Time of Total Delay. The most intuitional evaluation index of evaluating signal timing scheme is vehicle delay time, and the research shows that [19] fuel consumption, emission, and parking number are the same in the trend of change as optimization indexes. Hence, the minimum value of the total vehicle delay in the traffic network at the intersections is taken as the objective function can be given by

$$
J_{1}=\sum_{k} \sum_{t} \sum_{i} \sum_{j} D_{i j}^{k}(t)
$$

(b) Traffic Density. In the condition of multi-intersections information interchange, the traffic density is also the objective of regulating signal control, and it can be used as another objective function under traditional situation of minimum total delay. And it can be given by function (20).

$$
J_{2}=\max \left(\rho_{i}^{k}(t)\right)
$$

where $\rho_{i}^{k}(t)$ is the instantaneous traffic density of the $i$ traffic flow of intersection $k$ at time $t$, which can be obtained by

$$
\rho_{i}^{k}(t)= \begin{cases}\frac{\left(N_{i 2}(t)-Q_{i 3, g}(t)\right)}{L_{i}^{k}}, & \text { green } \\ \frac{N_{i 2}(t)}{L_{i}^{k}} & \text { red, }\end{cases}
$$

where $L_{i}^{k}$ is the length of the road corresponding to the $i$ traffic flow at the $k$ intersection. The other symbols are the same as formula (5).

\section{(ii) Restrictions}

(a) Cycle Time. According to the traffic volume of the $k$ intersection to set cycle time $T^{k}$, according to psychological impact of the driver, a single phase time should be not too short or too long; it can be constrained as expression (24).

$$
T_{\min }<T^{k}<T_{\max }, \quad\left(T_{\min }=50 \mathrm{~s}, T_{\max }=240 \mathrm{~s}\right) .
$$

(b) Split Ratio. Where $g_{i}^{k}$ is green time of the $i$ phase at the $k$ intersection, $r_{i}^{k}$ is red light time, $y^{k}$ is the yellow light time, and the split ratio $\lambda_{i}^{k}$ is shown as

$$
\begin{aligned}
g_{i}^{k}+r_{i}^{k}+y^{k} & =T^{k}, \\
\lambda_{i}^{k}=\frac{g_{i}^{k}}{T^{k}}, & \\
& \quad g_{\min }<g_{i}^{k}<g_{\max },
\end{aligned}
$$

where $g_{\min } \geq 15 \mathrm{~s}, g_{\max } \leq 120 \mathrm{~s}$ are the maximum and minimum green time.

(c) Phase Difference. Taking Figure 3 as an example, intersection sequence number ascending order is defined as uplink and descending order is defined as downlink; the $k-1$ intersection is the base intersection, so the uplink and downlink phase differences show constraint relationship as shown in (26).

$$
\theta^{k, k-1}+\theta^{k-1, k}=n T^{k}, \quad 0 \leq \theta^{k-1, k} \leq T^{k},
$$




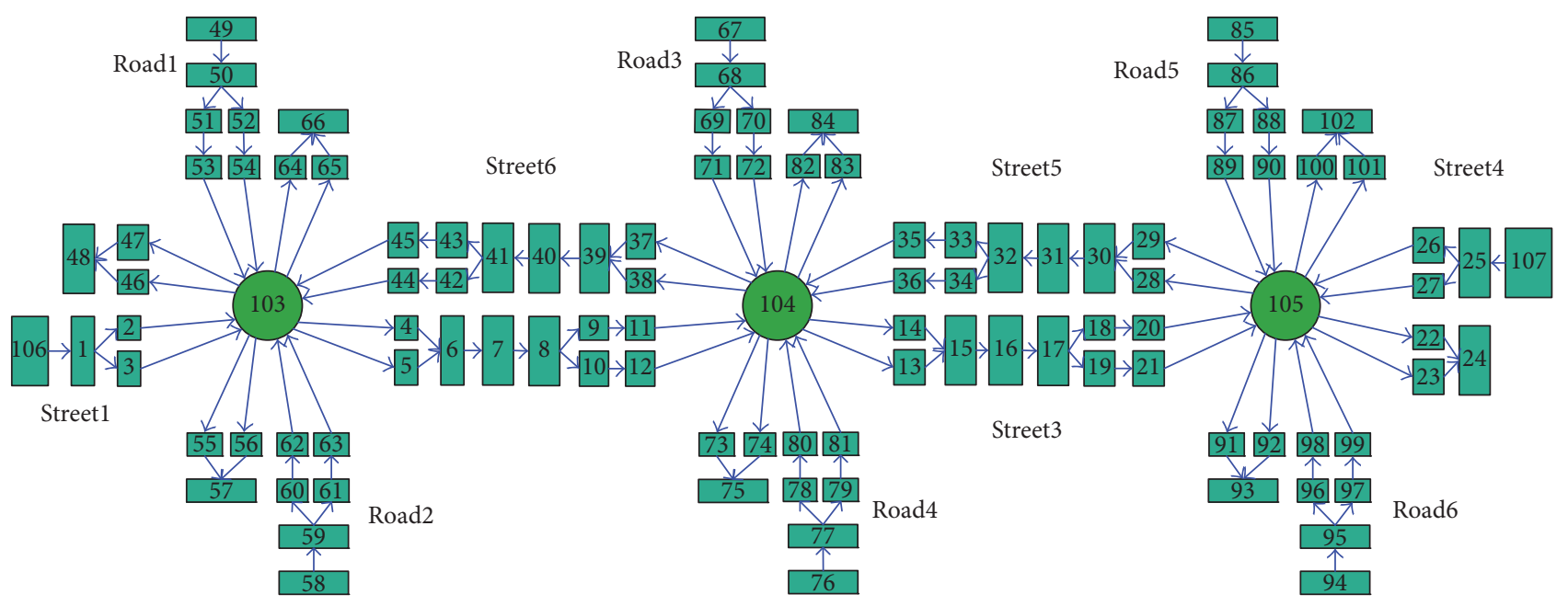

FIGURE 4: Multi-intersection simulation model based on Visual Disco.

where $\theta^{k-1, k}$ is the phase difference between the $k-1$ and $k$ intersection, and $n$ is an integer. For adjacent intersections, there is a need for cooperative signal control. So there is a cofactor $\delta^{k-1, k}$, when $\delta^{k-1, k}=1$ indicates phase synchronization of adjacent intersections.

(d) Capacity of Vehicles of a Section Road. The intersection signal coordination control must ensure that the control section does not appear as queue overflow, so the capacity of vehicles in the intersections is also a constraint; the current traffic volume $q_{i}^{k}$ should be calculated as in

$$
q_{i}^{k}<Q_{i, \max }^{k}=\bmod \frac{m \cdot L_{i}^{k}}{l_{c}} .
$$

Equation (27) shows that the maximum traffic capacity $Q_{i \text {,max }}^{k}$ of a road is related to the road length $L_{i}^{k}$ and the number of lanes $m$ and the road space $l_{c}$ occupied by a single vehicle.

3.3. Improved GA Used for Multi-Intersection. According to analysis of the model of multi-intersection road network based on CTM model in Section 2.2, it can be seen that the traffic volume is adjusted by the signal cycle $T^{k}$ and green time $\left\{g_{1}^{k}, g_{2}^{k}, \ldots, g_{n}^{k}\right\}$ and the phase differences $\left\{\theta_{i}^{k, k+1}, \theta_{i}^{k, k+2}, \ldots, \theta_{i}^{k, k+m}\right\}$ and so on between the intersections. In this paper, take the minimum delay $D_{i j}^{k}(t)$ as the objective function $J$ of the optimal control, where green light time and the phase difference between adjacent intersections were selected as optimization variables. This is shown in

$$
F=f\left(\left\{g_{1}^{k}, g_{2}^{k}, g_{3}^{k}, g_{4}^{k}\right\},\left\{\theta^{k-1, k}, \theta^{k, k+1}\right\}\right)
$$

In function (28), for a single intersection, the green time optimization parameter is four variables $\left\{g_{1}^{k}, g_{2}^{k}, g_{3}^{k}, g_{4}^{k}\right\}$ and the phase difference parameter is two variables $\left\{\theta^{k-1, k}, \theta^{k, k+1}\right\}$, so there are 18 optimization parameters of three intersections with four-phase signals. Obviously to satisfy realtime requirements, improved CTM needs to introduce the optimization algorithm.

Genetic algorithm (GA) is a search algorithm in computational mathematics, which is used to solve the optimization problem and it is a kind of evolutionary algorithm. The optimization of signal control for multi-intersections is a NP hard problem. As the number of optimized intersections increased, the search space becomes bigger. Hence, if the standard genetic algorithm (SGA) is used for multiobjective optimization directly, the convergence speed is slow in the optimization process, and the process will bring the system that is difficult to converge or the occurrence of premature convergence, which choose parameters of initialized, crossover, and fitness function arbitrarily and without the prior knowledge. So we selected an improved GA in [20], which is used for optimization problem with same prior knowledge needs. Under the same initial value, compared with the standard genetic algorithm optimization, for firstand fourth-order binary function $F_{1}(s)=21.5+x_{1}$. $\sin \left(4 \pi x_{1}\right)+x_{2} \sin \left(20 \pi x_{2}\right)$ and $F_{2}(s)=100\left(x_{1}^{2}-x_{2}\right)^{2}+\left(1-x_{1}\right)^{2}$, the improved genetic algorithm achieves $62 \%$ of the SGA in the search time, and the search efficiency is improved about more than 3 times of the effectiveness of the improved algorithm.

\section{Numerical Simulation and Result Analysis}

The Visual Disco simulation software (DISCO) was used for trunk road traffic simulation, which is developed by Lo [21], Hong Kong University, for the problem of optimization of minimum delay.

4.1. Model of Multiple Intersections Based on DISCO. The structure model of traffic flow through the intersection based on DISCO was shown in Figure 4, which is shown in Figure 2. Get the intersection signal time in simulation by the real 
signal timing of the environment. The input traffic flow was corresponding to the real situation, and it is set by manual work; the traffic flow state parameters are obtained from the virtual detector in the software. The traffic flow state parameters are used for signal optimization, which is according to the improved CTM model above and genetic algorithm (GA). Put the optimized signal timing into the road network to obtain the optimized minimum delay curve and traffic flow density curve, and it is compared with current curve controlled by real signal timing.

The rounded nodes with numbers in Figure 4 represent the intersection numbers; a rectangle with a number represents a cell whose width represents the number of lanes. For example, the cell width of number 1 indicates three lanes, cell 2 indicates one lane, and cell 3 indicates two lanes. According to the location of the intersection, we used the vehicle delay of upstream section of an intersection as a study object. Figure 3 described a local area network, the east-west direction consists of six sections, Street $1, \ldots$, Street6, and the north-south is Road $1, \ldots$, Road2. Specific composition is shown in Table 1. The first cell of an intersection upstream is defined as the signal-controlled cell. Junction line between two cells indicates the flow direction of traffic; if the cells are constituted with divergence or confluence, which flow from the upstream into downstream cells, the traffic value of divergence or confluence flow is computed according to the ratio of $p_{i}, p_{c}$ in (9), (10).

4.2. Parameters Initialization. For the DISCO model established in this paper, the simulation time is set as 1000 seconds. The delay of vehicles in the road was recorded, which is shown in Figure 2, and the delay time is used as the optimization objective function in unit time; we capture the output of simulation results interval of 40 seconds. Because the rightturn vehicle is not under the signal control, assume that there is no effect on the control process but it exists on the rightturn traffic. The initial phase signal control timing scheme is shown in Table 1.

For the traffic volume in the separated cell, setting the proportion of turning left and right and going straight to total traffic volume is $15 \%, 15 \%$, and $70 \%$, and going straight and turning left vehicles are controlled. For the simulation of local road network in Figure 5, set the saturated traffic flow rate is $Q_{\max }=1800 \mathrm{vel} / \mathrm{h}$, blocking density is $\rho_{\text {jam }}=125 \mathrm{vel} / \mathrm{km}$, and the relationship between mean flow and vehicle density can be found in STCA-S model from Figure 6 [17]; road speed limit is $V_{\max } \approx 20 \mathrm{~m} / \mathrm{s}=72 \mathrm{~km} / \mathrm{h}$. The amount of traffic data varies with the simulation timing as shown in Table $1 . C_{\min }=$ 1458 vehicles/h, and $C_{\max }$ value is calculated by Figure 7 from literature [11] Figure 18(e).

4.3. Results and Analysis. The initial traffic density was set as 0.2 . The traffic flow of every source cell of Street $1, \ldots$, Street 6 and Road $1, \ldots$, Road 2 is determined in Table 2, in which traffic flow value is changed according to $200 \mathrm{~s}$ as a cycle. Numerical simulations results are carried out by the signal setting method of fixed timing and improved CTM. The optimal method of SGA and improved GA was compared
TABLE 1: Intersection time initialization.

\begin{tabular}{lcc}
\hline Intersection & Phase & Time (second) \\
\hline 103 & Phase 1 & 50 \\
104 & Phase 2 & 15 \\
105 & Phase 3 & 40 \\
& Phase 4 & 15 \\
\hline
\end{tabular}

in the same situation. The length of every road is set as real situation shown in Table 3 .

(i) Minimum Delay Time. The average delay volume is calculated by different method of time setting. The traffic flow volume of source cell was changed in different periods, which is shown in Table 2, and the curve of minimum delay time of each intersection varies with the input traffic volume as shown in Figure 5.

In Figure 5, the cyan-blue curve is the normalized change of input traffic volume by source cell at each intersection; other color curves meanings were shown in subframe of Figure 5. Compared with the delay time results of fixed timing and SGA and improved GA in (a), (b), (c), it shows that, in the fixed timing plan, the average delay of the vehicle changes with the traffic volume. The delay time of each intersection increases rapidly when the input traffic volume exceeds $720 \mathrm{vel} / \mathrm{h}$. The red curve and black curve both are simulation results of improved CTM with different optimal method; it shows that the signal timing plan is changed by improved CTM, which can reduce the effects of increased input traffic volume for delay time. Compared with the average delay variation curve obtained by SGA, the optimization GA method is similar to the trend of the curve change, but the average delay is lower; it shows that the improved GA introduced in this paper has better real-time performance than SGA and shows that a rapid calculating method can better adapt to the change of traffic flow.

Under conditions discussed in Section 3.1, capacity of intersection is related with the density of upstream traffic flow. From the purple curve "improved GA and CTM" in Figure 5, we found that, at simulation time 400 seconds, the average delay time was still increased with a small slope, which is not similar as curve "improved GA"; vehicles may have to wait with random delay at the downstream stop line even with $\bar{q}_{\text {in }}<C_{\min }$. This is due to the fact that the turning right flow is uncontrolled in the upstream intersection. Hence, when both the flows of cell ${ }_{49}$ and cell ${ }_{58}$ are increased, the probability of synchronized flow becomes high and the delay time is increased. From $400 \mathrm{~s}$ to $600 \mathrm{~s}$, the slope of average delay time curve changes slowly. Because of the $t$ synchronized flow condition, compared with $600 \mathrm{~s}$, the value of intersection 103 density is lower than $580 \mathrm{~s}$, implying the efficiency of our timing plan. After $600 \mathrm{~s} \bar{q}_{\text {in }}>C_{\max }$, the delay time should increase with traffic flow. However, from the subfigure (a) we can see that, from $600 \mathrm{~s}$ to $800 \mathrm{~s}$, the delay time is controlled with more green phases time for trunk line.

From the curves in Figures 5(a), 5(b), and 5(c) at simulation time section $400 \mathrm{~s}-800 \mathrm{~s}$, densities of "improved 

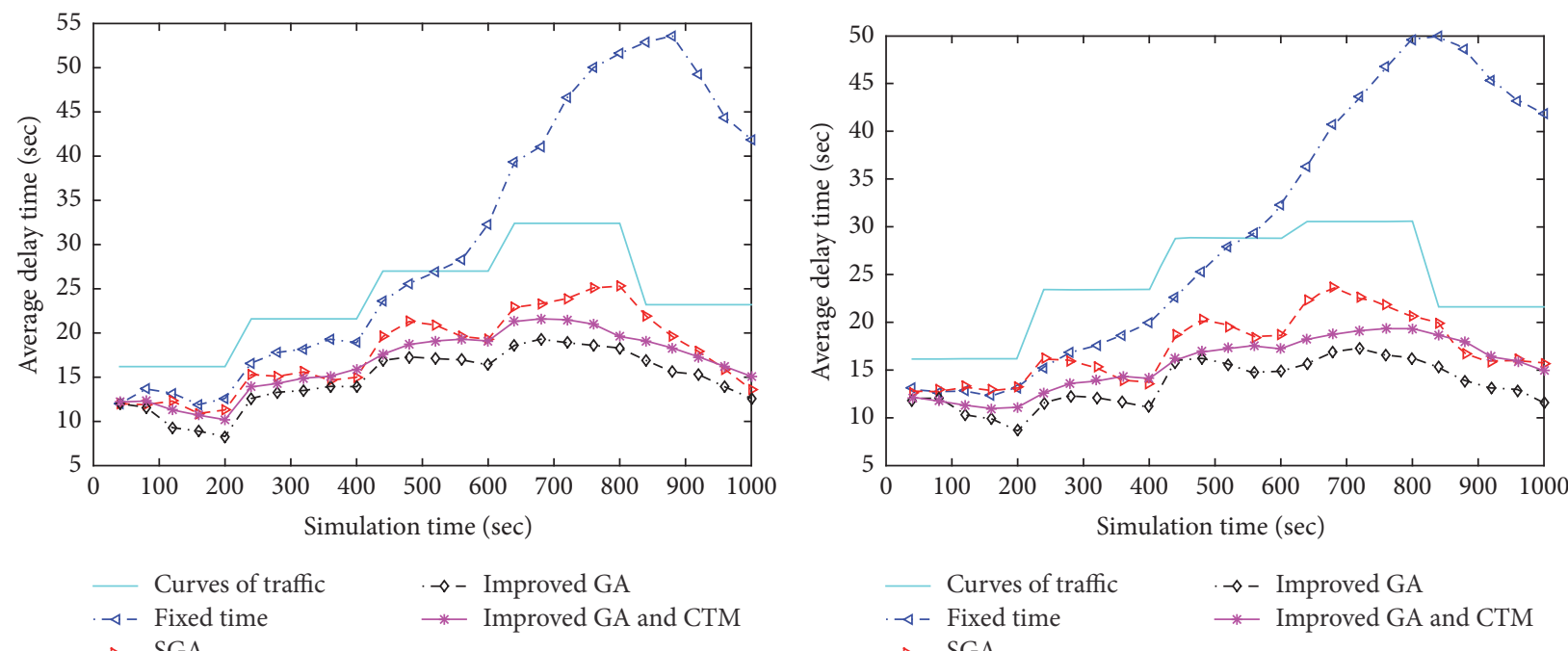

(a) Intersection 103

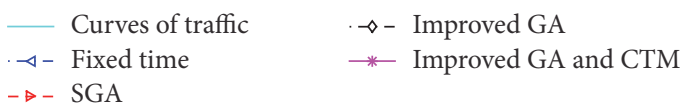

(b) Intersection 104
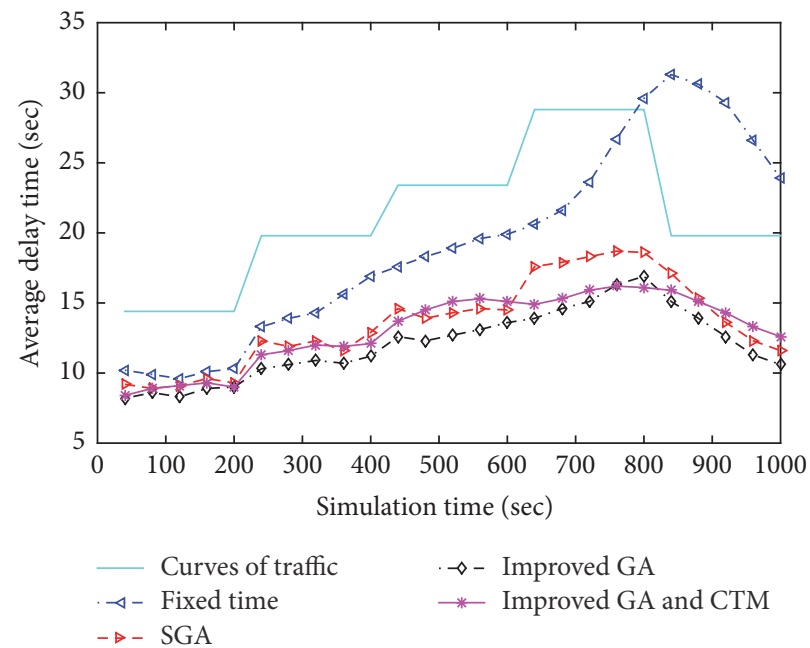

(c) Intersection 105

FIGURE 5: Curve of average delay of each intersection.
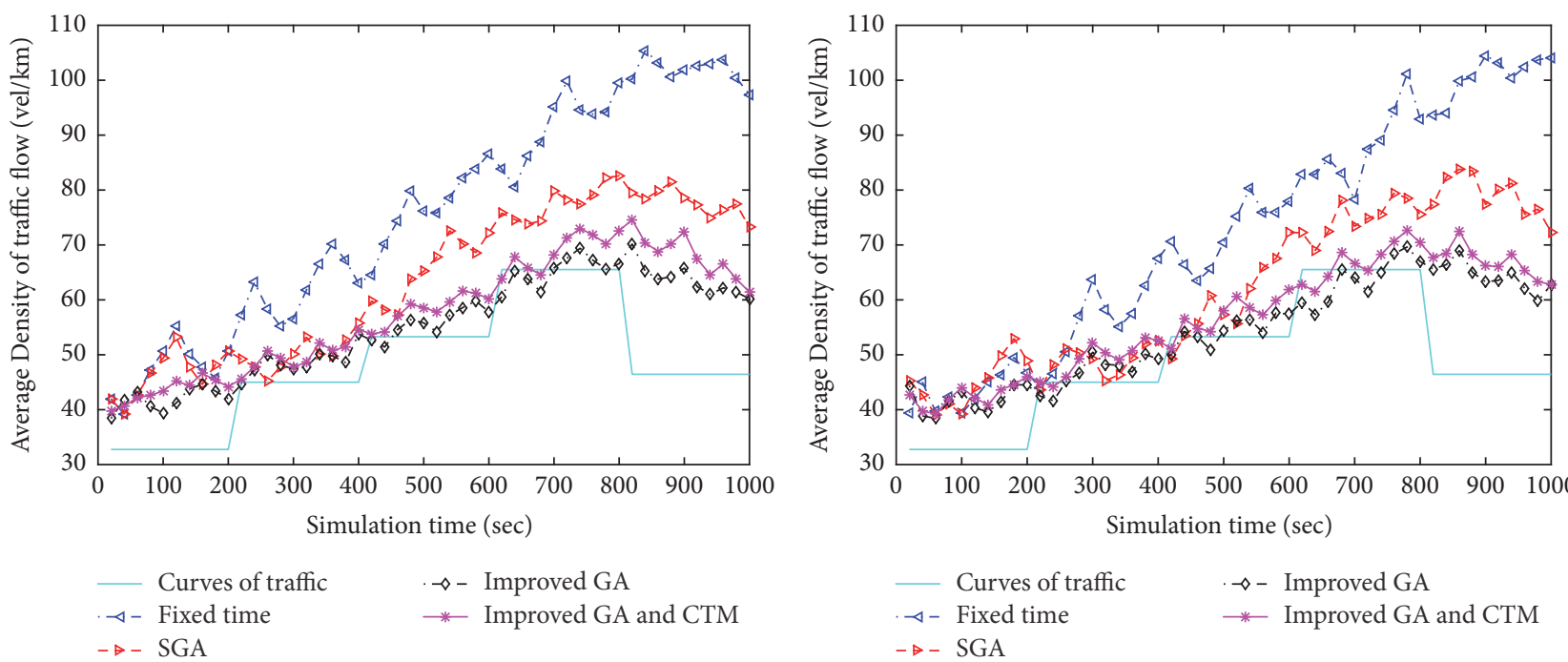

(a) Street 2

(b) Street3

Figure 6: Curve of road traffic density and simulation time. 
TABLE 2: Setting of traffic input value.

\begin{tabular}{|c|c|c|c|c|c|}
\hline Source cells & $0 \sim 199 \mathrm{~s}$ & $200 \sim 399 \mathrm{~s}$ & $400 \sim 599 \mathrm{~s}$ & $600 \sim 799 \mathrm{~s}$ & $800 \sim 999 \mathrm{~s}$ \\
\hline cell $_{106}$, cell $_{107}$ & $900 \mathrm{vel} / \mathrm{h}$ & $1260 \mathrm{vel} / \mathrm{h}$ & $1440 \mathrm{vel} / \mathrm{h}$ & $1800 \mathrm{vel} / \mathrm{h}$ & $1260 \mathrm{vel} / \mathrm{h}$ \\
\hline ceell $_{49}$, cell $_{58}$ & $720 \mathrm{vel} / \mathrm{h}$ & $900 \mathrm{vel} / \mathrm{h}$ & $1260 \mathrm{vel} / \mathrm{h}$ & $1440 \mathrm{vel} / \mathrm{h}$ & $1080 \mathrm{vel} / \mathrm{h}$ \\
\hline cell $_{67}$, cell $_{76}$ & $720 \mathrm{vel} / \mathrm{h}$ & $1080 \mathrm{vel} / \mathrm{h}$ & $1440 \mathrm{vel} / \mathrm{h}$ & $1260 \mathrm{vel} / \mathrm{h}$ & $900 \mathrm{vel} / \mathrm{h}$ \\
\hline cell $_{85}$, cell $_{94}$ & $540 \mathrm{vel} / \mathrm{h}$ & $720 \mathrm{vel} / \mathrm{h}$ & $900 \mathrm{vel} / \mathrm{h}$ & $1080 \mathrm{vel} / \mathrm{h}$ & $720 \mathrm{vel} / \mathrm{h}$ \\
\hline
\end{tabular}

TABLE 3: Length of road setting.

\begin{tabular}{lccccccc}
\hline Name of road & Street1 & Street $2 / 6$ & Street3/5 & Street4 & Road $1 / 2$ & Road3/4 & Road5/6 \\
\hline Length of road $(\mathrm{m})$ & 1100 & 776 & 600 & 670 & 860 & 900 & 900 \\
\hline
\end{tabular}

TABLE 4: Comparison table of the average delay value.

\begin{tabular}{lcccccc}
\hline \multirow{2}{*}{ Timing plan } & \multicolumn{7}{c}{ Sampling (s) } \\
& No. & 200 & 400 & 600 & 800 & 1000 \\
\hline \multirow{3}{*}{ Fixed } & $103 \#$ & 12.67 & 18.79 & 32.33 & 51.61 & 41.92 \\
& $104 \#$ & 13.16 & 19.91 & 32.36 & 49.63 & 41.22 \\
& $105 \#$ & 10.56 & 16.83 & 20.03 & 29.39 & 23.78 \\
\hline \multirow{3}{*}{ SGA } & $103 \#$ & 11.35 & 14.99 & 19.63 & 25.53 & 13.76 \\
& $104 \#$ & 13.13 & 13.69 & 18.76 & 20.59 & 15.77 \\
& $105 \#$ & 9.37 & 12.89 & 14.46 & 18.67 & 11.93 \\
\hline \multirow{3}{*}{ Improved GA } & $103 \#$ & 8.32 & 13.93 & 16.69 & 18.33 & 12.69 \\
& $104 \#$ & 8.61 & 11.13 & 14.89 & 16.37 & 11.86 \\
& $105 \#$ & 8.97 & 11.25 & 13.62 & 16.73 & 10.65 \\
Improved GA with & $103 \#$ & 10.21 & 15.93 & 19.13 & 19.62 & 15.16 \\
improved CTM & $104 \#$ & 11.12 & 14.13 & 17.25 & 19.31 & 15.07 \\
& $105 \#$ & 9.07 & 12.11 & 15.19 & 16.16 & 12.03 \\
\hline
\end{tabular}

GA and CTM" are higher than that of "improved GA," because the effect of traffic flow density for the intersection capacity is considered based on the three-phase theory. In particular, when traffic flow approaches or exceeds the value of GW breakdown threshold, the intersection capacity then is significantly reduced, and the control model thus needs to improve the situation with the increase of green timing.

From the subfigures (a), (b), and (c), when the traffic flow volume is increased at simulation time $(200 \mathrm{~s}, 400 \mathrm{~s}$, and $600 \mathrm{~s})$, the data of Table 4 shows that, compared with the SGA, the average delay time of improved GA is reduced to $34.57 \%$ and the minimum value is only $15.06 \%$ in the case of lower input traffic volume $\left(q_{\text {in }} \leq 900 \mathrm{vel} / \mathrm{h}\right)$; when the input traffic volume increased $\left(q_{\text {in }} \geq 1260 \mathrm{vel} / \mathrm{h}\right)$, the average delay time of improved GA is reduced as maximum $21.02 \%$ and minimum $30.05 \%$, and the absolute value of the average delay time is reduced to 7 seconds.

The simulation results of the improved CTM by threephase theory show that, at lower input traffic volume, the average delay time is higher than the improved GA by $17.3 \%$, a result caused by the random behaviors of right turning vehicles. With a large traffic flow, the average delay time also grows faster under GW control for the intersection capacity limited and the delay time is higher than that of the improved GA about $20.05 \%$ (1000 s) in maximum value and $9.1 \%$ (400s), 14.4\% (600 s), and 16.13 (800s) in minimum values. Simulation data indicate that when the traffic flow is in synchronous phase, optimization control method of this paper has better effect.

At the same time, compared with the average delay curve of the corresponding high-input traffic volume in Figure 5, due to shared traffic flow information of each intersection, the method of improved CTM in this paper can perform cooperative control of the signals, which can adjust each intersection signal timing scheme according to the variation of upstream and downstream traffic flow, especially when the traffic volume is higher. The average delay curve obtained from improved CTM is gentle and the absolute value is low. Therefore, it can be seen that the improved GA can be used to reduce the delay of vehicles and improve the capacity of trunk road by the optimized intersections.

(ii) Traffic Density. Taking the traffic density of the two representative sections Street 2 and Street 3 as the examples, the traffic density curve between the change of traffic input and signal control under different control methods is drawn in Figure 6.

From Figure 6(a) we can see that as the traditional timing distribution scheme does not have the vehicle traffic density changes in adaptive control, the street 2 traffic density curve has a regular fluctuation with the traffic light. And when the input traffic increases, the traffic density increases for the fixed timing scheme lack of adaptability. Under the SGA optimization mode, the timing scheme changes with the road traffic density, so the fluctuation trend change in the traffic volume is small.

However, because of the real-time requirement, the optimal value obtained by SGA in the same search time is inferior to the improved method in this paper. So it did not show the best ability for the traffic changes. Because of the faster search capability, the improved GA can adjust the signal timing changes following the traffic flow by using the program better than SGA. So it shows obvious optimization ability in the overall section of the traffic volume. 
From the traffic density curve of street3 in Figure 6(b), we can see that the phase time changes with the input traffic volume. And because of the introduction of interactive information, comparing the curves and simulation time in (a), (b), we can see that while the street 3 traffic volume changes, the signal light phase differences of 104 and 103 intersections are optimized to adapt to the changes of road traffic density. So the traffic density under this method makes the red-green phase change gently. We can see the multi-intersection coordination control method in the road which is mentioned in this paper, which can consider the traffic volume and the timing scheme of the intersections comprehensively. The improved GA can search the optimal value faster than the SGA. So the optimization effect is better than the other two signal control methods and has certain coordination and optimization capabilities for the number of intersections of the signal timing program

In addition to the above analysis, the curve of "improved GA and CTM" shows that tendency of the density is the same as the "improved GA" curve, but the value of density is different with "improved GA." Before simulation time $400 \mathrm{~s}$, because the speed of vehicles passing the intersection of both models is free flow speed, the numerical difference between the two curves is tiny. Difference is mainly derived from uncontrolled turning in vehicles. From simulation time $400 \mathrm{~s}-700 \mathrm{~s}$, synchronized flow is increased with more vehicles intakes, and the green wave GW is unstable, which can be seen from the graph, where the slope of "improved GA and CTM" density curve is greater than "improved GA." At green light time section, the slope of "improved GA and CTM" density curve is less than "improved GA." Compared with improved GA, number of crossed vehicles is decreased. Summarize the analysis above, due to the employment of the three-phase traffic flow, the traffic density of road section is higher in our improved CTM, which has less crossing vehicles than that of the improved GA based on the same timing scheme.

\section{Conclusion}

This paper presents an improved CTM for adjusting the timing plan of multi-intersection on urban trunk road. The improved CTM is established based on the future information infrastructure with interactive conditions. The intersections of Youyi Road were chosen as collaborative optimization object, and the improved GA is used for multiobjective optimization, which is according to the realtime requirements of traffic control. Finally effectiveness of improved CTM is demonstrated by numerical simulation based on DISCO software. By the simulation, the timing plans of fixed timing, SGA, improved GA were compared. Simulation results show that, based on the CTM, the dynamic characteristics of traffic flow can be simulated. Under the situation of improved CTM, intersection signal timing plan can be according to the variation of upstream and downstream traffic and the adaptive timing plan can be provided.

By the adaptive timing plan based on improved CTM, the signal cooptimization control model is established, which can reduce the influence of delay time as traffic volume changed.
At the same time, comparing the simulation results of SGA and improved GA and improved CTM, it is shown that improved GA is is more rapid than SGA for multiobjective optimization, and it can supply intersection signal timing plan with better adaptability, but the improved CTM is more suitable with the actual traffic flow controlled situation, in which vehicles are moving among intersections of trunk road. According to improved CTM in this paper, optimizing the signal timing at intersections on trunk road, it can control traffic density indirectly and play a role to improve the efficiency of road use.

\section{Conflicts of Interest}

The authors declare that they have no conflicts of interest.

\section{Acknowledgments}

This research is subsidized by The Natural Science Basic Research Plan in Shaanxi Province of China under Grant no. 2016JQ5106; Dr. Startup Funds of Xian Polytechnic University (no. BS1507); Special Research Program Funded by Shaanxi Provincial Education Department (16JK1342); and Discipline Construction Funds of Xian Polytechnic University under Grant no. 107090811.

\section{References}

[1] S. Zhongke, Q. Shiru, H. Huixian et al., Introduction to Traffic Control System, Science Press, Beijing, China, 2003.

[2] K. Mucsi, Adaptive Urban Bimodal (Car and Transit) Transportation System Management in Saturated and Oversaturated Conditions, Carleton University, Ontario, Canada, 2009.

[3] B. Park, C. Messer, and T. Urbanik II, "Enhanced genetic algorithm for signal-timing optimization of oversaturated intersections," Transportation Research Record: Journal of the Transportation Research Board, vol. 1727, pp. 32-41, 2000.

[4] B. Park, C. Messer, and T. Urbanik, "Traffic signal optimization program for oversaturated conditions: a genetic algorithms approach," in Proceedings of the 78th Annual TRB Meeting, Washington, Wash, USA, January 1999.

[5] E. Lieberman and J. Chang, "Optimizing traffic signal timing through network decomposition," Transportation Research Record, vol. 1925, no. 1, pp. 167-175, 2005.

[6] G. Abu-Lebdeh and R. F. Benekohal, "Design and evaluation of dynamic traffic management strategies for congested conditions," Transportation Research Part A: Policy and Practice, vol. 37, no. 2, pp. 109-127, 2003.

[7] M. J. Lighthill and G. B. Whitham, "On kinematic waves. II. A theory of traffic flow on long crowded roads," Proceedings of the Royal Society: London A: Mathematical, Physical and Engineering Sciences, vol. 229, pp. 317-345, 1955.

[8] A. H. F. Chow and H. K. Lo, "Sensitivity analysis of signal control with physical queuing: delay derivatives and an application," Transportation Research Part B: Methodological, vol. 41, no. 4, pp. 462-477, 2007.

[9] W. Zhengwu, L. Dayong, and H. Zhongxiang, "Optimization designing and solving of signal based on CTM," Journal of Traffic and Transportation Engineering, no. 4, pp. 84-89, 2007. 
[10] G. Yunfeng, H. Hua, H. Hao et al., "Multi-objective optimization and simulation of urban road intersection group signal coordination control," China Journal of Highway and Transport, no. 6, pp. 129-136, 2012 (Chinese).

[11] B. S. Kerner, "Three-phase theory of city traffic: moving synchronized flow patterns in under-saturated city traffic at signals," Physica A: Statistical Mechanics and Its Applications, vol. 397, no. 3, pp. 76-110, 2014.

[12] B. S. Kerner, S. L. Klenov, and M. Schreckenberg, "Traffic breakdown at a signal: classical theory versus the three-phase theory of city traffic," Journal of Statistical Mechanics: Theory \& Experiment, vol. 2014, no. 3, p. 126, 2014.

[13] C. F. Daganzo, "The cell transmission model, part II: network traffic," Transportation Research B, vol. 29, no. 2, pp. 79-93, 1995.

[14] Y. Dongxia and J. Yongfeng, "Multi-objective optimization method of traffic signal based on CTM," Journal of Traffic and Transportation Engineering, no. 3, pp. 105-111, 2011 (Chinese).

[15] Urban Road Design Code, People's Republic of China industry standard (CJJ 37-90).

[16] B. S. Kerner and S. L. Klenov, "Phase transitions in traffic flow on multilane roads," Physical Review E, vol. 80, no. 5, Article ID 056101, 2009.

[17] L. Xun, Q. Shiru, and X. Yu, "Coperative lane-changing rules on multilane under condition of cooperative vehicle and infrasture system," China Journal of Highway and Transports, vol. 27, no. 8, pp. 97-104, 2014.

[18] B. S. Kerner, "The physics of green-wave breakdown in a city," Europhysics Letters, vol. 102, no. 2, Article ID 28010, 2013.

[19] Y. Xiaoguang, Z. Jing, M. Wanjing et al., "Study on the computation method of signal control intersection capacity," China Journal of Highway and Transport, no. 5, pp. 150-156, 2014 (Chinese).

[20] X. Li, S. Qu, and L. Cai, "Studing of PID parameter optimization method for attitude controller of quadrotor," Journal of Computational Information Systems, vol. 10, no. 18, pp. 7805-7812, 2014.

[21] H. K. Lo, "A novel traffic signal control formulation," Transportation Research Part A: Policy and Practice, vol. 33, no. 6, pp. 433-448, 1999. 


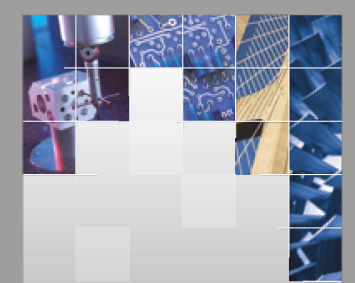

\section{Enfincering}
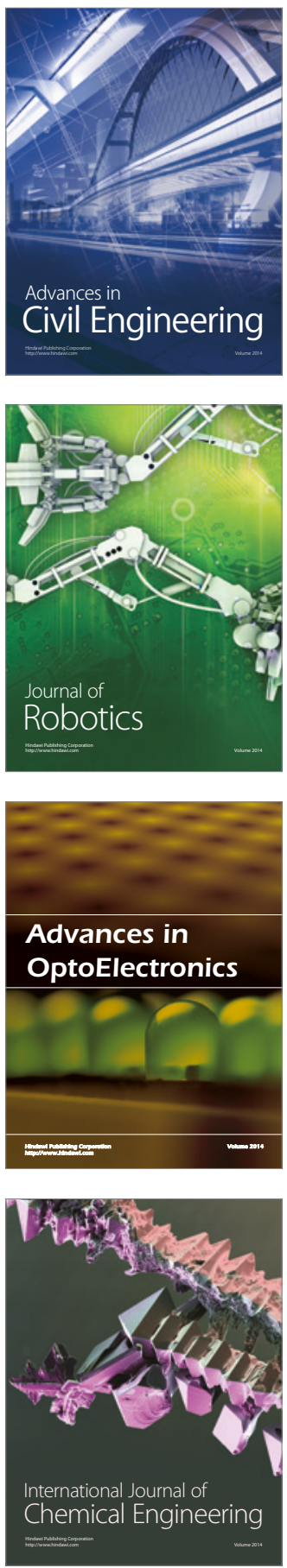

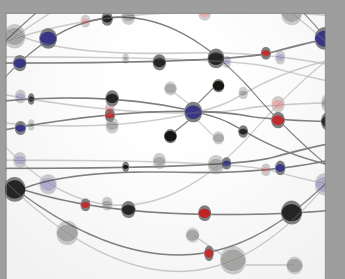

The Scientific World Journal

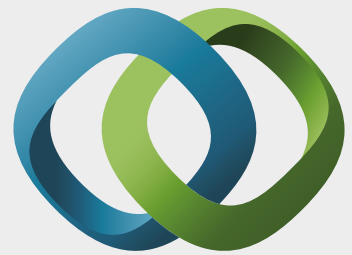

\section{Hindawi}

Submit your manuscripts at

https://www.hindawi.com
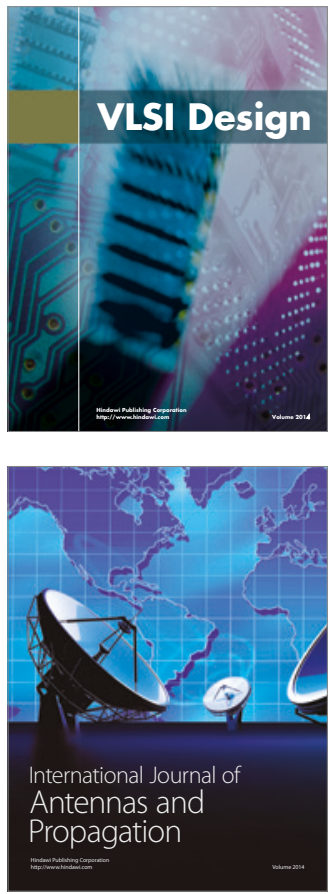

\section{Rotating}

Machinery
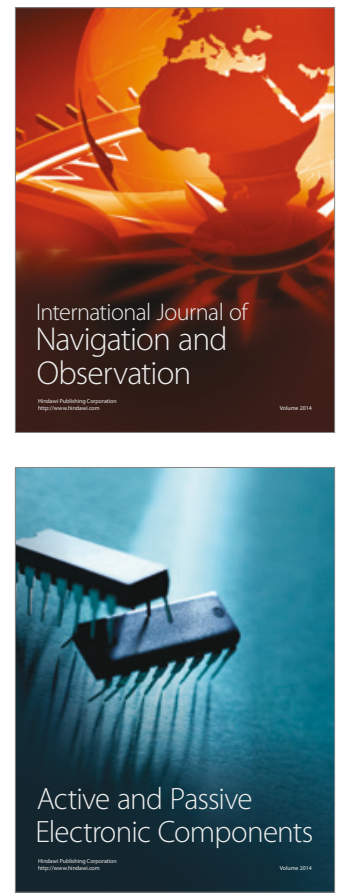
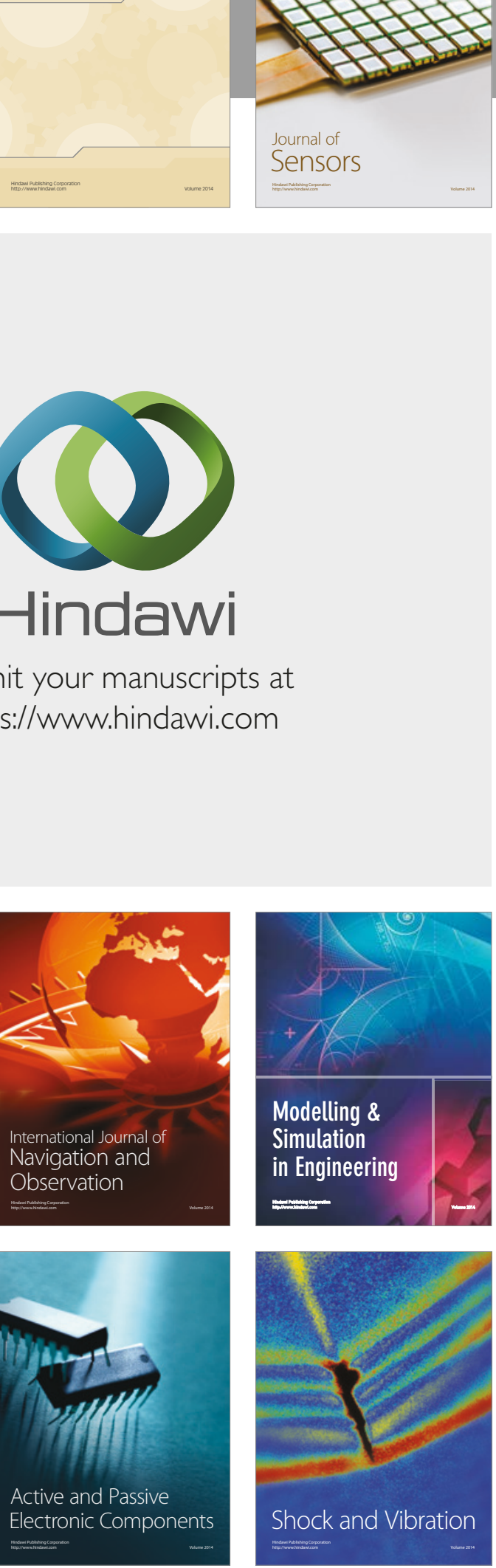
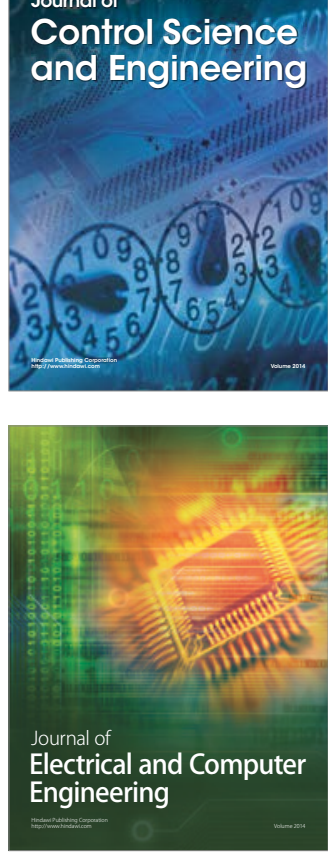

Distributed

Journal of

Control Science

and Engineering
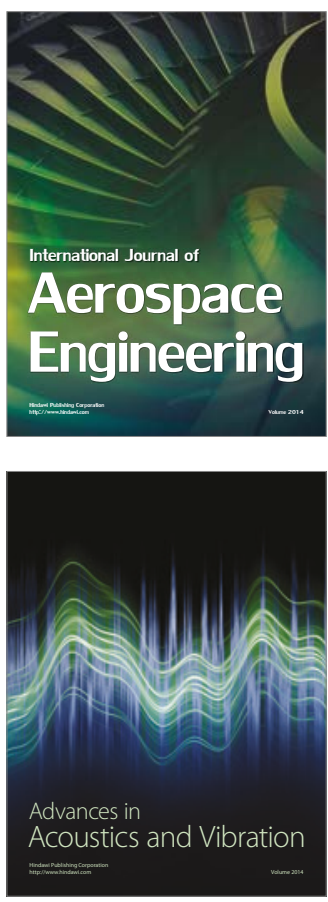

Sensor Networks 